\title{
IDH1 Mutation Induces HIF-1 $\alpha$ and Confers Angiogenic Properties in Chondrosarcoma JJ012 Cells
}

\author{
Xiaoyu Hu, ${ }^{1,2}$ Luyuan Li, ${ }^{3,4}$ Josiane E. Eid, ${ }^{3,4}$ Chao Liu, ${ }^{2}$ Jinming Yu $\mathbb{D}^{1,2}{ }^{1,2}$ Jinbo Yue $\mathbb{D}^{2}$ \\ and Jonathan $C$. Trent ${ }^{3,4}$ \\ ${ }^{1}$ Department of Oncology, Renmin Hospital of Wuhan University, Wuhan, China \\ ${ }^{2}$ Department of Radiation Oncology, Shandong Cancer Hospital and Institute, Shandong First Medical University and Shandong \\ Academy of Medical Sciences, Jinan, Shandong, China \\ ${ }^{3}$ Department of Medicine, Division of Medical Oncology, University of Miami Miller School of Medicine, Miami, USA \\ ${ }^{4}$ Sylvester Comprehensive Cancer Center, University of Miami Miller School of Medicine, Miami, USA
}

Correspondence should be addressed to Jinming Yu; sdyujinming@163.com, Jinbo Yue; jbyue@sdfmu.edu.cn, and Jonathan C. Trent; jtrent@med.miami.edu

Xiaoyu $\mathrm{Hu}$ and Luyuan Li contributed equally to this work.

Received 12 September 2021; Accepted 15 December 2021; Published 14 February 2022

Academic Editor: Ting Su

Copyright (c) 2022 Xiaoyu Hu et al. This is an open access article distributed under the Creative Commons Attribution License, which permits unrestricted use, distribution, and reproduction in any medium, provided the original work is properly cited.

\begin{abstract}
Chondrosarcoma is a group of primary bone cancers that arise from transformed cells of chondrocytic lineage. Tumor recurrence and metastasis are devastating for patients with chondrosarcoma since there are no effective treatment options. IDH mutations occur in over $50 \%$ of tumors from patients with conventional or dedifferentiated chondrosarcomas and represent an attractive target for therapy. However, their role in the pathogenesis of chondrosarcoma remains largely unknown. In this study, we sought to determine the association of IDH mutation and HIF- $1 \alpha$ in chondrosarcoma. We used the chondrosarcoma JJ012 cell line and its derived CRISPR/Cas9 mutant IDH1 (IDH1 ${ }^{\text {mut }}$ ) knockout (KO) cells. RNA-Seq data analysis revealed downregulation of several HIF- $1 \alpha$ target genes upon loss of IDH1 ${ }^{\text {mut }}$. This was associated with reduced HIF- $1 \alpha$ levels in the $\mathrm{IDH}_{1}{ }^{\text {mut }} \mathrm{KO}$ cells and tumors. Loss of IDH1 $1^{\text {mut }}$ also attenuated the expression of angiogenic markers in tumor tissues and abrogated the angiogenic capacity of JJ012 cells. Moreover, we observed that exogenous expression of HIF-1 $\alpha$ significantly promoted anchorage-independent colony-formation by $\mathrm{IDH} 1^{\text {mut }} \mathrm{KO}$ cells. These results suggest IDH1 mutation confers angiogenic and tumorigenic properties of JJ012 cells by inducing HIF-1 $\alpha$. Thus, the HIF pathway represents a promising candidate for combinatorial regimens to target IDH1 mutated chondrosarcomas.
\end{abstract}

\section{Introduction}

Chondrosarcomas constitute a heterogeneous group of primary bone cancers characterized by the formation of a hyaline cartilaginous matrix. Following osteosarcoma, chondrosarcoma is the second most common bone malignancy, accounting for $20 \%$ to $27 \%$ of primary bone tumors $[1,2]$. Approximately $85 \%$ of chondrosarcomas are the conventional subtypes which can be further classified into central, peripheral, and periosteal lesions. The remaining $10-15 \%$ consist of rare subtypes including dedifferentiated, mesenchymal, clear cell, and myxoid chondrosarcoma. Chondrosarcomas are notoriously resistant to chemotherapy and radiotherapy, and surgery is the backbone treatment for most localized tumors $[2,3]$. Chondrosarcomas tend to recur with more aggressive behavior than the original neoplasm following initial tumor resection. As a result, many patients develop metastatic disease which is nearly uniformly fatal. Due to lack of effective treatment strategies for recurrent or metastatic chondrosarcoma, high-grade conventional and dedifferentiated chondrosarcomas have poor prognosis $[1,4]$. Current studies focus on clarifying the link between molecular events and pathogenesis of this malignancy and developing new molecularly targeted therapies for advanced diseases. 
Isocitrate dehydrogenase (IDH) mutation is among one of the promising therapeutic targets. IDH1/2 mutations were found in $71 \%$ of conventional chondrosarcomas and $57 \%$ of dedifferentiated chondrosarcomas $[4,5]$, as well as in gliomas and acute myeloid leukemia $[6,7]$, suggesting a potential role for aberrant IDH function in the pathogenesis of these malignancies. IDHs normally convert isocitrate to $\alpha$ ketoglutarate $(\alpha-\mathrm{KG})$. However, mutant IDHs lose the ability to catalyze this reaction but instead gain a neomorphic function of reducing $\alpha$-KG to D-2-hydroxyglutarate (D-2HG), which has been reported to accumulate at high levels in IDH1/2-mutated tumors $[8,9]$. D-2HG and $\alpha$-KG are structurally similar. Thus, accumulated D-2HG is thought to act as an oncometabolite through the inhibition of various $\alpha$ KG-dependent enzymes including the TET family of 5methycytosine hydroxylases, JumonjiC domain-containing histone demethylases (JHDMs), and the Prolyl Hydroxylase Domain-Containing Proteins (PHDs) [9-11].

HIF- $1 \alpha$, a key hypoxia-inducible transcription factor, is associated with tumor development as it functions as a master regulator of genes involved in angiogenesis, glucose metabolism, and other cellular pathways [12]. HIF-1 $\alpha$ overexpression is correlated with disease progression, chemoradio-resistance, and increased patient mortality in certain cancers [13-15]. The stability and transcriptional activity of HIF- $1 \alpha$ are regulated by PHDs (PHD1, PHD2, and PHD3). Under normal oxygen conditions, PHDs utilize $\alpha$ $\mathrm{KG}$ and $\mathrm{O}_{2}$ to hydroxylate a conserved proline in HIF- $1 \alpha$, leading to Von Hippel-Lindau- (VHL-) mediated ubiquitination and subsequent proteasomal degradation of HIF- $1 \alpha$ $[16,17]$. In hypoxic conditions, however, these hydroxylation events cannot proceed efficiently, resulting in accumulation of HIF-1 $\alpha$ [18]. Interestingly, studies examining the effects of D-2HG on PHDs and HIF- $1 \alpha$ in IDH-mutant gliomas and leukemias have yielded conflicting results. It has been reported that D-2HG competitively inhibits the activity of PHDs as mentioned above and thus leads to increased levels of HIF- $1 \alpha[18,19]$. Conversely, D-2HG was shown to act as an activator rather than an inhibitor of PHDs, ultimately leading to decreased levels of HIF-1 $\alpha$ [20, 21]. In contrast to the emerging knowledge in gliomas and leukemias, little is known regarding the effect of IDH mutation or D-2HG on HIF- $1 \alpha$ activity in chondrosarcoma. Understanding their relationship would have great clinical importance in terms of developing novel targeted therapies for advanced chondrosarcomas.

In this study, we sought to determine the potential association of IDH mutation and HIF- $1 \alpha$ in chondrosarcoma. We employed the IDH1-mutant chondrosarcoma JJ012 cell line, CRSPR/Cas9 mutant IDH1 (IDH1 ${ }^{\text {mut }}$ ) knockout (KO) JJ012 clones, and their derived xenografts. We found that CRISPR/Cas9 knockout of IDH $1^{\text {mut }}$ reduced HIF- $1 \alpha$ levels in vitro and in vivo, leading to downregulation of HIF- $1 \alpha$ target genes. Loss of IDH $1^{\text {mut }}$ also decreased the expression of angiogenic markers in tumors and attenuated the angiogenic capacity of JJ012 cells. Moreover, we observed restoring HIF- $1 \alpha$ levels with exogenous expression significantly enhanced the anchorage-independent growth of $\mathrm{IDH} 1^{\mathrm{mut}}$ KO cells. These results suggest IDH1 mutation confers tumorigenic and angiogenic properties by inducing HIF- $1 \alpha$ in a JJ012 chondrosarcoma model.

\section{Materials and Methods}

2.1. Cell Culture. The human chondrosarcoma JJ012 and human chondrocyte C28 cell lines were kindly provided by Dr. Joel Block and Dr. Karina Galoian, respectively. JJ012 harbors a monoallelic IDH1 R132G mutation while C28 carries wildtype IDH1 (IDH1 ${ }^{\mathrm{wt}}$ ). JJ012 cells were cultured in RPMI-1640 medium (Lonza) supplemented with $10 \%$ fetal bovine serum (FBS) and 1\% Penicillin/Streptomycin. C28 cells were grown in 1:1 DMEM/F12 medium (HyClone) supplemented with 10\% FBS and 1\% Penicillin/ Streptomycin. Human umbilical vein endothelial cells (HUVECs) were obtained from Thermo Fisher Scientific and grown in Endothelial Cell Growth Medium 2 (EGM-2) (PromoCell) on $0.1 \%$ gelatin-coated plates. Cells were maintained at $37^{\circ} \mathrm{C}$ in a humidified air with $5 \% \mathrm{CO}_{2}$.

2.2. IDH1 Knockout by CRISPR/Cas9 Technology. Knockout of IDH $1{ }^{\text {mut }}$ was achieved by the CRISPR/Cas9 system. The CRISPR/Cas9 plasmid products were purchased from Santa Cruz Biotechnology. Details of transfection, selection, and single-cell colonies propagation were previously described [22].

2.3. Measurement of D-2HG. Quantitative analyses of D$2 \mathrm{HG}$ were conducted by high-performance liquid chromatography-tandem mass spectrometry (HPLC-MS/MS) with single-reaction monitoring (SRM) scans. This was performed at MtoZ Biolabs (Boston, MA, USA).

2.4. Tumor Tissues. Tumor samples were obtained from mice-bearing chondrosarcoma xenografts which were derived from JJ012 parental and IDH $1^{\text {mut }} \mathrm{KO}$ cells, as previously described. All animal experiments were performed in compliance with the University of Miami Institutional Animal Care and Use Committee (IACUC)-approved protocol (No. 19-079).

2.5. RNA-Seq and Ingenuity Pathway Analysis (IPA). RNASeq and IPA were described with details previously [22].

2.6. Quantitative Reverse Transcriptase Polymerase Chain Reaction ( $q R T-P C R)$. Total RNA was extracted using the miRNeasy Mini Kit (Qiagen) according to the manufacturer's protocol. cDNA was synthesized from $2 \mu \mathrm{g}$ of total RNA using iScript a cDNA synthesis kit (Bio-Rad:1708891) in a $40 \mu \mathrm{l}$ total volume. qRT-PCR was set up with 20x TaqMan probes, $2 \mu \mathrm{l}$ of 1:5 diluted cDNA and TaqMan universal PCR Master Mix (Thermo Fisher Scientific) in $20 \mu \mathrm{l}$ total volume. Samples were run in triplicate on a Bio Rad CFX-96 real time PCR system. Gene expression levels were calculated using the $2^{-\Delta \Delta \mathrm{Ct}}$ method [23]. Gene-specific TaqMan primers/probe sets include GAPDH (internal normalization control), VEGFA, VEGFC, EDN1, and SLC2A3.

2.7. Western Blotting. Cells were lysed in a Laemmli sample buffer (Bio-Rad) supplemented with 2-mercaptoethanol (Sigma-Aldrich). The lysates were centrifuged at 
$14,000 \mathrm{rpm}$ for $10 \mathrm{~min}$ at $4^{\circ} \mathrm{C}$. The supernatants were collected and denatured at $95^{\circ} \mathrm{C}$ for $10 \mathrm{~min}$. Equal protein lysates were separated on 4-20\% Mini-PROTEIN TGX precast gels (Bio-Rad) and transferred to nitrocellulose membranes (Pall Corporation). The following antibodies were used: anti-IDH1 (1:1000, Abcam, ab172964), anti-HIF-1 $\alpha$ $(0.5 \mu \mathrm{g} / \mathrm{ml}$, RD Systems, NB100-105), and anti- $\beta$-actin (1:5000, Cell Signaling).

2.8. Soft-Agar Colony Formation Assay. $5 \times 10^{3}$ cells were plated in a $0.3 \%$ top layer soft agar in RPMI-1640 with $10 \%$ FBS overlaid on a lower layer of a $0.5 \%$ basal agar. Cells were then maintained in regular incubator with $21 \% \mathrm{O}_{2}$, or in a hypoxia chamber with $1 \% \mathrm{O}_{2}$ at $37^{\circ} \mathrm{C}$ for $10-14$ days. Colonies were stained with $2 \mathrm{mg} / \mathrm{mL}$ iodonitrotetrazolium chloride (Sigma-Aldrich), rinsed with PBS, and quantified with GelCount colony counter (Oxford Optronix).

2.9. Immunohistochemistry (IHC). Immunohistochemical analysis was performed on $5-\mu \mathrm{m}$ sections cut from formalin-fixed, paraffin-embedded samples utilizing antibodies against CD31 (Servicebio, GB11063-2), HIF-1 $\alpha$ (Bioworld, BS3514), VEGFA (Abcam, ab52917), and IDH1 (Abcam, ab172964) following a standard protocol. A semiquantitative evaluation method was applied as follows: the score obtained by the percentage of positive cells $(0 \%=0 ; 1-25 \%=1,26$ $-50 \%=2,51-75 \%=3$, and $>75 \%=4$ ) was multiplied by the score obtained by the staining intensity (no staining $=0$ , weak staining $=1$, moderate staining $=2$, and strong staining $=3$ ). Scoring was evaluated by investigators who were blinded to the information of research subjects.

2.10. Vascular-Endothelial Tube Formation Assay. JJ012 parental and IDH1 $1^{\text {mut }} \mathrm{KO}$ cells were cultured with serumsupplemented RPMI-1640 overnight, followed by a $24 \mathrm{~h}$ starvation in serum-free medium. Secretome derived from the culture supernatant was then collected and centrifuged at $1200 \mathrm{rpm}$ for $5 \mathrm{~min}$ to remove debris. The vascularendothelial tube formation assay was performed with HUVEC cells in 24-well plates pre-coated with growth factor-reduced basal membrane Geltrex matrix (Thermo Fisher Scientific) and left to solidify for $30 \mathrm{~min}$ at $37^{\circ} \mathrm{C}$. HUVEC cells were harvested with Trypsin/EDTA solution and the cell concentrations were determined in nonsupplemented EGM-2. $2 \times 10^{5}$ cells were seeded in each well and incubated with $1 \mathrm{ml}$ of cell supernatant secretome for $24 \mathrm{hr}$. After incubation, cells were stained with Calcein AM $(2 \mu \mathrm{g} / \mathrm{ml})$ for $30 \mathrm{~min}$, rinsed with $1 \mathrm{mM} \mathrm{CaCl}_{2}$ and $0.5 \mathrm{mM}$ $\mathrm{MgCl}_{2}$-supplemented PBS, and fixed with $4 \%$ paraformaldehyde in PBS for $25 \mathrm{~min}$ at room temperature. Capillary tubes were visualized with fluorescence microscopy. The total vascular-endothelial tube lengths were measured from randomly selected image fields per sample per group using $\mathrm{NIH} /$ Image J. At least five fields per well were examined, and each experimental condition was tested in triplicate. All HUVEC cells used in experiments underwent fewer than eight passages after resuscitation.

2.11. Statistical Analysis. GraphPad Prism 8 software was used for statistical analyses. $p$ values were determined by unpaired, two-tailed $t$ tests. $p<0.05$ was considered statistically significant.

\section{Results}

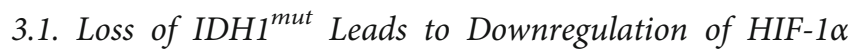
Target Genes in JJ012 Cells. In this study, we utilized the human chondrosarcoma JJ012 cell line which harbors an endogenous IDH mutation, and its derived two CRISPR/ Cas9 IDH $1^{\text {mut }} \mathrm{KO}$ clones. Depletion of the IDH1 protein and reduced $\mathrm{D}-2 \mathrm{HG}$ levels in the two $\mathrm{IDH} 1^{\text {mut }} \mathrm{KO}$ clones are shown in Figures 1(a) and 1(b), respectively. To begin to understand the role of IDH mutation in chondrosarcoma tumorigenesis, we initially conducted an RNA-Seq analysis of JJ012 parental cells and its two IDH $1^{\text {mut }} \mathrm{KO}$ clones. The RNA-Seq analysis revealed an association between IDH mutation and aberrant activation of integrin signaling in chondrosarcoma [22]. Interestingly, in addition to mediators of cell adhesion and integrin-related pathways, we found in the transcriptome of JJ012 IDH $1^{\text {mut }} \mathrm{KO}$ cells many genes known to be involved in vasculogenesis. The downregulated HIF- $1 \alpha$ target genes detected in both IDH $1{ }^{\text {mut }} \mathrm{KO}$ clones are shown in Figure 1(c). These genes are implicated in glucose metabolism (SLC2A3, LDHA and LDHB) and angiogenesis (VEGFA and VEGFC). Downregulation of several wellestablished HIF- $1 \alpha$ target genes including VEGFA, VEGFC, EDN1 and SLC2A3 in the IDH1 ${ }^{\text {mut }} \mathrm{KO}$ cells was further confirmed by qRT-PCR (Figure $1(\mathrm{~d})$ ). These results indicate that IDH mutation is associated with activation of the HIF$1 \alpha$ signaling pathway.

To be noted, both $\mathrm{IDH}_{1}{ }^{\mathrm{wt}}$ and $\mathrm{IDH}_{1}{ }^{\text {mut }}$ were knocked out in our cell model. To confirm that it is the loss of IDH1mut allele rather than that of $\mathrm{IDH} 1^{\mathrm{wt}}$ is responsible for the downregulation of the HIF- $1 \alpha$ target genes, we utilized C28 cells, an immortalized human chondrocyte cell line that expresses IDH $1^{\mathrm{wt}}$ only [24]. A pool of C28 IDH $1^{\mathrm{wt}} \mathrm{KO}$ cells with markedly reduced IDH1 levels was created using the CRISPR/Cas9 technique (Figure 1(e)). qRT-PCR analysis revealed comparable expression of the aforementioned HIF- $1 \alpha$ target genes between C2 8 parental and IDH $1^{\mathrm{wt}} \mathrm{KO}$ cells (Figure $1(\mathrm{f})$ ), suggesting that loss of the $\mathrm{IDH}_{1}{ }^{\text {mut }}$ rather than of the IDH $1^{\text {wt }}$ allele caused the downregulation of the HIF- $1 \alpha$ target genes in JJ012 cells.

3.2. Loss of IDH $1^{\text {mut }}$ Reduces HIF-1 $\alpha$ Levels in JJ012 Cells and Tumor Tissues. HIF- $1 \alpha$ protein levels are regulated by PHDs which destabilize the angiogenic transcription factor by post-translational proline hydroxylation under normoxic conditions. It has been reported that D-2HG competitively inhibits PHDs due to its structural similarity to $\alpha-\mathrm{KG}$, thereby causing accumulation of HIF- $1 \alpha$ in IDH1-mutant glioma cells [19]. In our model, D-2HG production was almost completely suppressed in JJ012 IDH1 ${ }^{\text {mut }} \mathrm{KO}$ clones [22]. Therefore, it is rational to inquire whether the downregulation of HIF- $1 \alpha$ target genes in the IDH $1{ }^{\text {mut }} \mathrm{KO}$ cells is attributed to HIF- $1 \alpha$ inhibition as a result of $\mathrm{IDH} 1^{\text {mut }}$ loss and reduced D-2HG production. Indeed, HIF- $1 \alpha$ expression appeared to be significantly decreased in the two IDH $1^{\text {mut }}$ KO JJ012 cell lines compared to their parental control under 


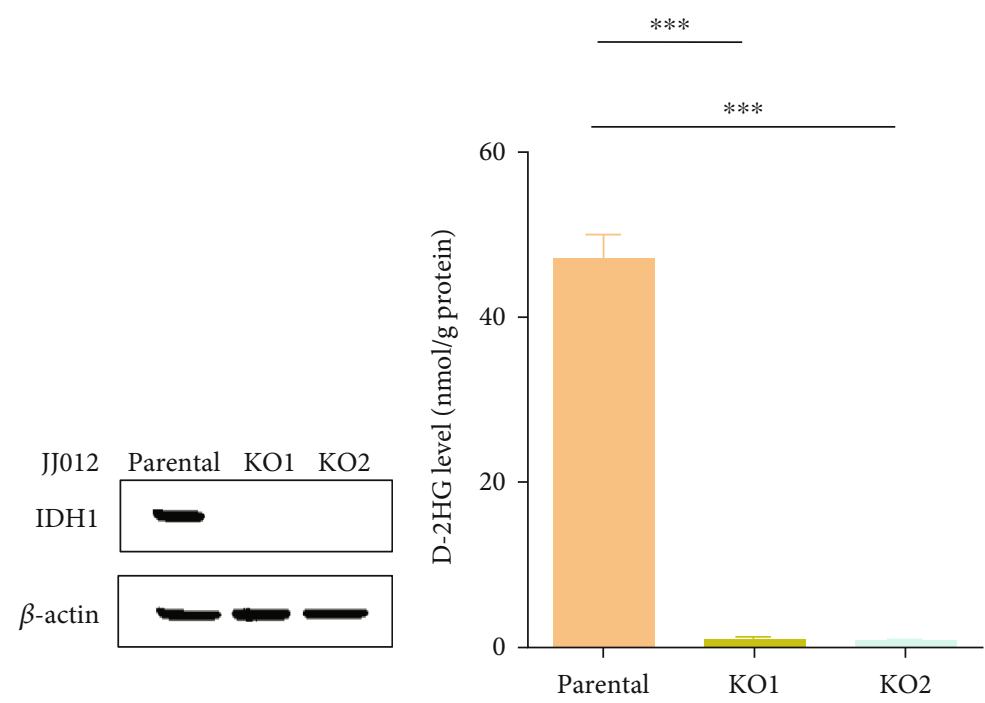

(a)

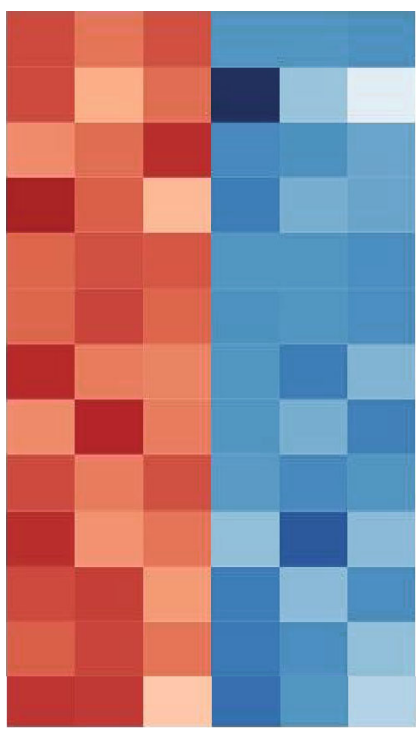

Parental

KO1

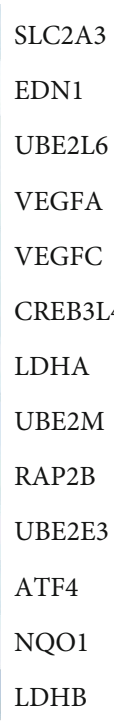

DHB

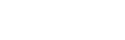

A3
2L6
GFA
E3L4
2B
2E 3

(b)

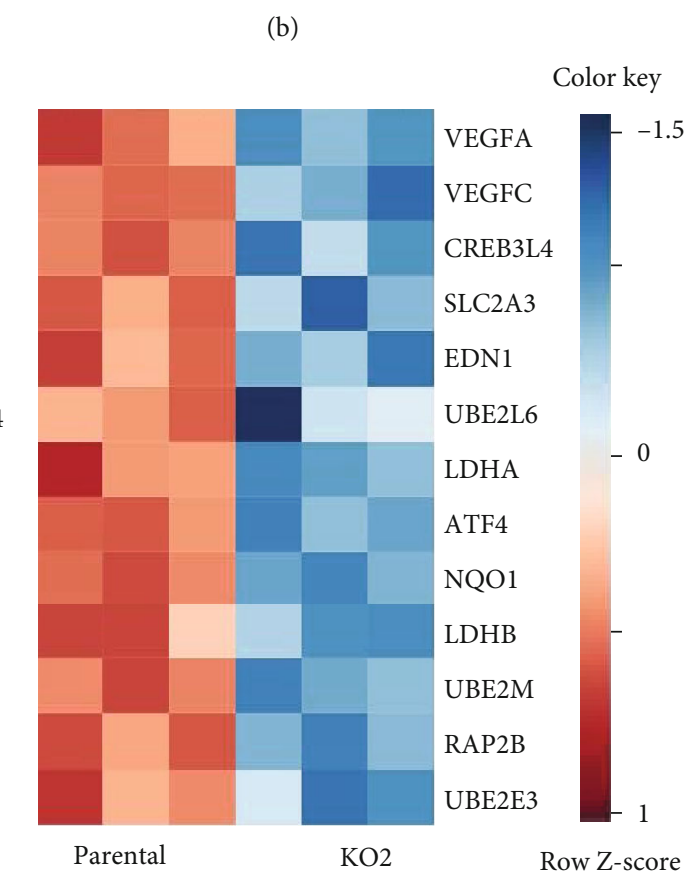

(c)

Figure 1: Continued. 

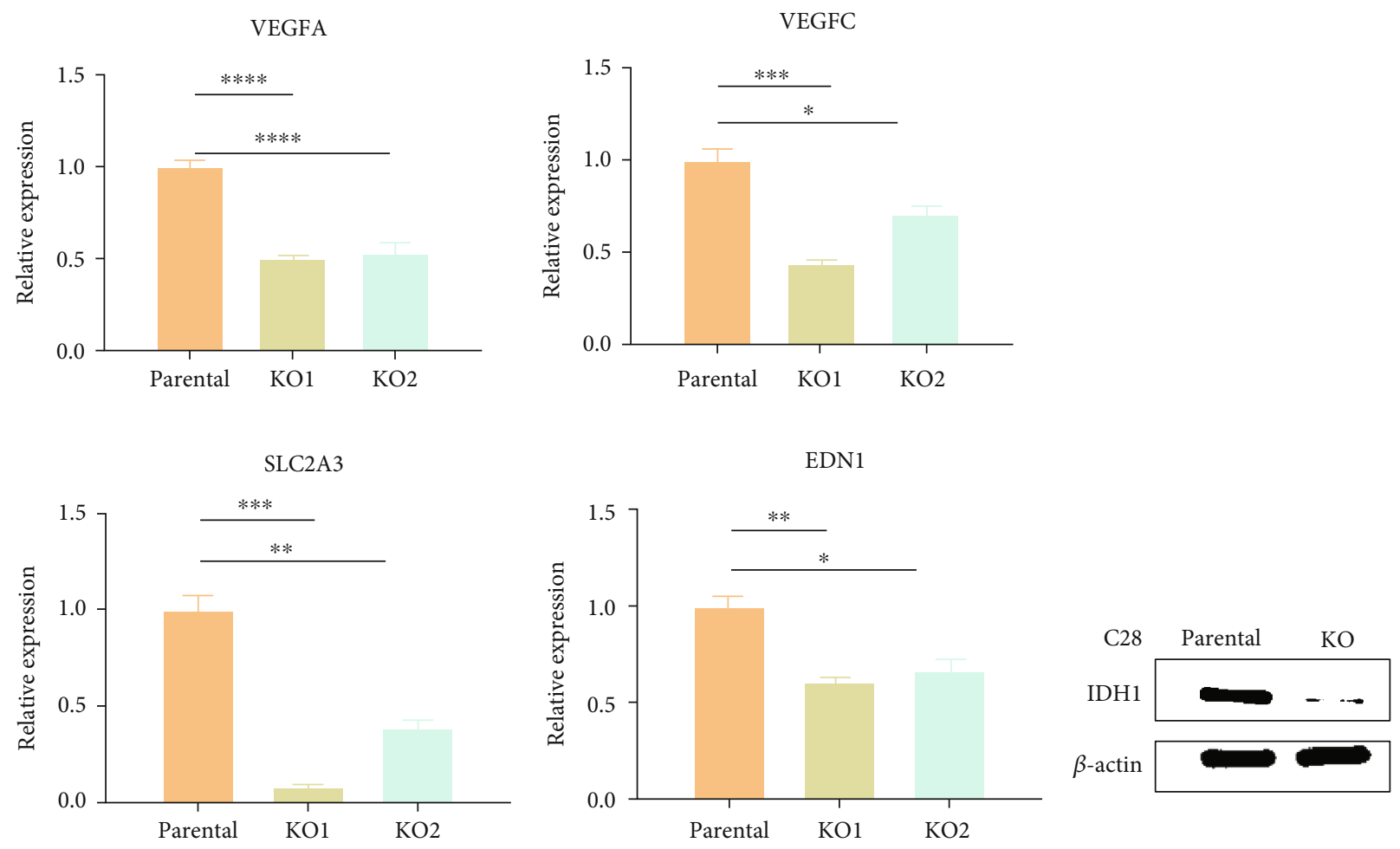

(d)

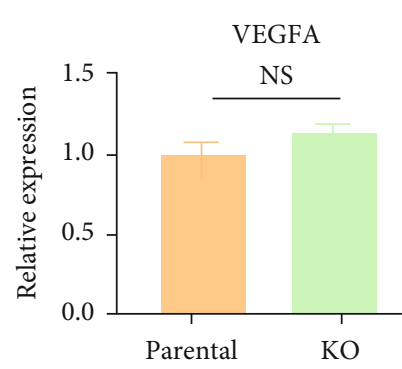

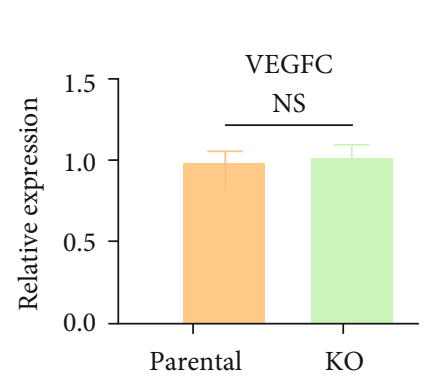

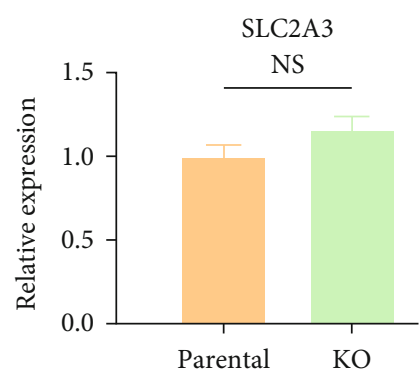

(f) (e)

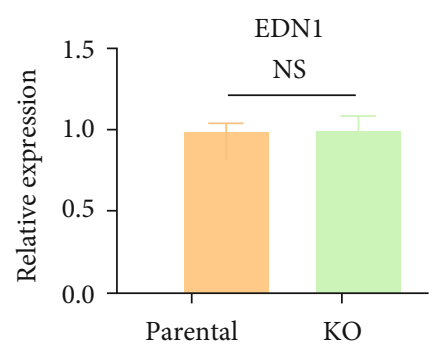

FIGURE 1: Downregulation of HIF- $1 \alpha$ target genes upon loss of IDH1 ${ }^{\text {mut }}$ in JJ012 cells. (a) Immunoblot shows depletion of IDH1 protein in two IDH $1{ }^{\text {mut }}$ KO clones of JJ012 cells. (b) HPLC-MS analysis indicates D-2HG production was nearly depleted in two IDH ${ }^{\text {mut }}$ KO clones of JJ012 cells. (c) Heatmaps of HIF- $1 \alpha$ target genes that are downregulated in both IDH1 ${ }^{\text {mut }} \mathrm{KO}$ clones compared to parental control of JJ012 cells. (d) mRNA expression of VEGFA, VEGFC, EDN1, and SLC2A3 in JJ012 cells was quantified by qRT-PCR. The amount of transcript was normalized to GAPDH, and the results are shown as fold-change relative to the parental control. Data are shown as mean $\pm \mathrm{SEM}$ of triplicate values and are representative of three independent experiments. $* p<0.05, * * p<0.01, * * * p<0.001$. (e) Immunoblot shows levels of wildtype IDH1 in CRISPR/Cas9 KO C28 chondrocytes. (f) Bar graphs compare levels of VEGFA, VEGFC, EDN1, and SLC2A3 mRNA expression in parental and wildtype IDH1 KO C28 cells. NS: nonsignificant $(p>0.05)$.

normoxic conditions (Figure 2(a)). We then proceeded to ask whether HIF- $1 \alpha$ was similarly regulated in vivo. For this we measured IDH1 expression in the chondrosarcoma xenografts derived from parental and IDH1 ${ }^{\text {mut }} \mathrm{KO} \mathrm{JJ} 012$ cells that we have previously established (22). IHC analysis revealed significant reduction of IDH1 in the IDH1 ${ }^{\text {mut }} \mathrm{KO}$ tumors (Figure 2(b)). Importantly, HIF- $1 \alpha$ levels were concomitantly reduced in the same tumors, thus confirming our in vitro findings. The staining score showed that HIF$1 \alpha$ expression was reduced by $70 \%-80 \%$ in the $\mathrm{IDH} 1^{\mathrm{mut}}$ KO tumors compared to parental controls (Figure 2(c)). These results demonstrate that knockout of IDH1 ${ }^{\text {mut }}$ downregulates HIF- $1 \alpha$ in vitro and in vivo, thus supporting the concept that IDH1 mutation promotes HIF- $1 \alpha$ stabilization and its downstream signaling in our JJ012 chondrosarcoma model.

\subsection{IDH1 Mutation Confers Angiogenic Properties in JJ012} Chondrosarcoma Cells. Angiogenesis represents an essential step in tumor proliferation, expansion, and metastasis, thus contributing to the pathology of virtually all human cancers. HIF- $1 \alpha$ is a subunit of HIF-1, an oxygen-dependent transcriptional activator, which plays crucial roles in tumor angiogenesis and mammalian development [12]. HIF-1 activates transcription of genes encoding angiogenic growth factors which are secreted by hypoxic cells and promote 


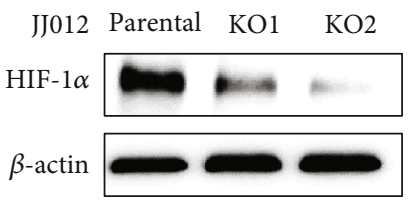

(a)
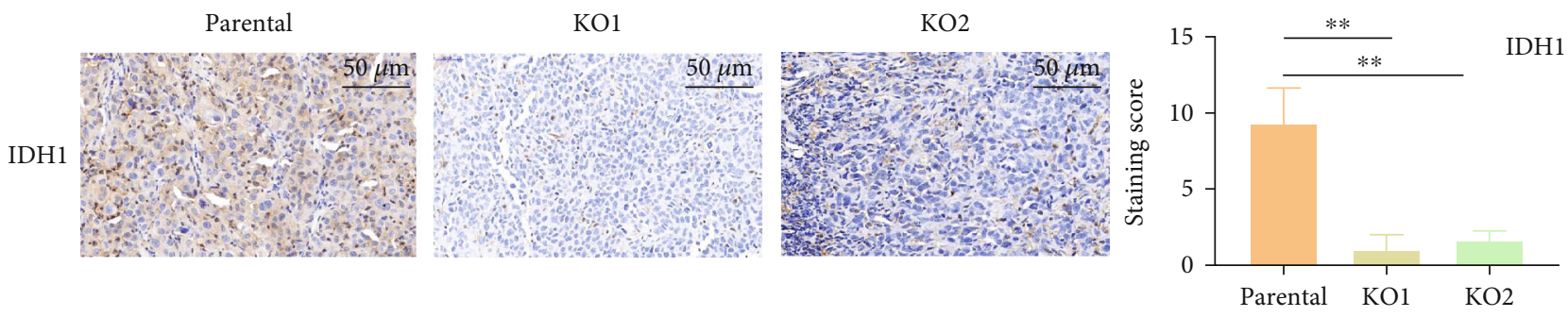

(b)
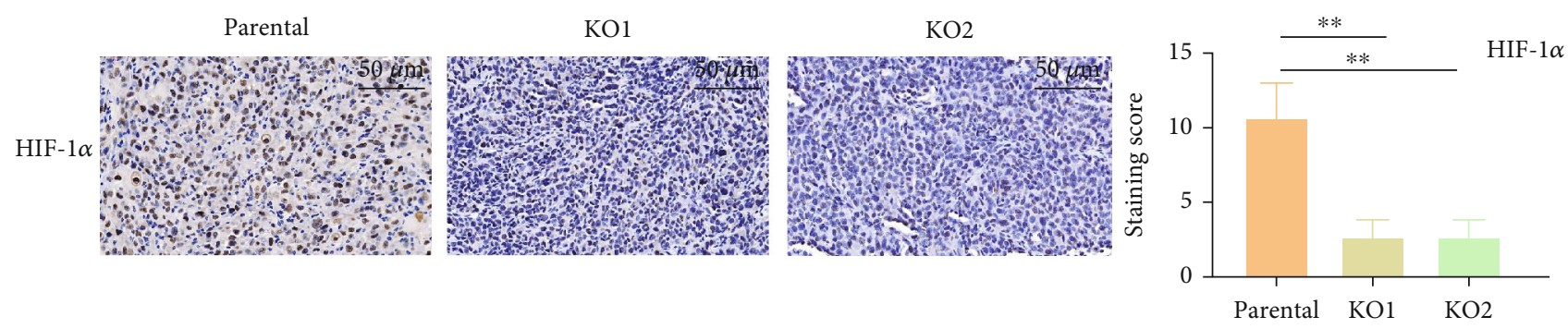

(c)

FIGURE 2: Loss of IDH1 ${ }^{\text {mut }}$ suppresses HIF- $1 \alpha$ in JJ012 cells and tumor tissues. (a) Immunoblots show HIF-1 $\alpha$ expression in JJ012 parental and IDH $1{ }^{\text {mut }} \mathrm{KO}$ cells; immunohistochemical (IHC) images show cytoplasmic IDH1 (b) and nuclear HIF-1 $\alpha$ (c) expression in parental and $\mathrm{IDH}_{1}{ }^{\text {mut }} \mathrm{KO}$ JJ012-derived xenografts. IHC staining was quantified with intensity scores as detailed in Materials and Methods. Scale bars are $50 \mu \mathrm{m}$. Data represent mean \pm SEM of values from four random fields. $* * p<0.01$.

endothelial cell growth [25]. Given the apparent association of HIF- $1 \alpha$ expression with IDH1 mutation, we asked whether such mutation confers angiogenic properties on the chondrosarcoma JJ012 cells. Interestingly, functional analysis of the transcriptome in JJ012 IDH1 ${ }^{\text {mut }} \mathrm{KO}$ cells identified angiogenesis as one of the most prominently regulated programs (adjusted $p=1.02 E-06$ ) in chondrosarcoma. Upon further examination of angiogenic markers by IHC, we observed that CD31 and VEGFA expression in JJ012 IDH1 ${ }^{\text {mut }} \mathrm{KO}$ cellderived tumors was markedly reduced when compared to parental cell-derived tumors (Figures 3(a) and 3(b)). These findings suggest a functional association of IDH1 mutation with the HIF- $1 \alpha$-driven angiogenic pathway. To further assess this functional link, we conducted vascular-endothelial tube formation assays using HUVECs incubated with the secretome of JJ012 parental or IDH1 ${ }^{\text {mut }} \mathrm{KO}$ cells. We found that culturing with the $\mathrm{DH} 1^{\text {mut }} \mathrm{KO}$ secretome significantly inhibited HUVECs ability to form vascular tubes and reduced capillary tube length by over $20 \%(p<0.05)$ (Figure $3(c))$. These findings indicate that IDH1-mutant JJ012 chondrosarcoma cells produce a secretome highly capable of stimulating angiogenesis and promoting tumor growth.

3.4. HIF-1 $\alpha$ Contributes to Tumorigenicity of IDH1 Mutation in JJ012 Cells. HIF-1 $\alpha$ regulates gene expression in critical pathways that drive tumorigenesis [25]. Thus, we endeav- ored to determine whether HIF- $1 \alpha$ contributes to the oncogenic properties of IDH mutation in the JJ012 chondrosarcoma cells. One of the defining criteria of tumorigenicity is anchorage-independent cell growth [26]. In our previous study, we demonstrated that loss of IDH1 ${ }^{\text {mut }}$ attenuated the tumorigenic potential of chondrosarcoma cells. In particular, depletion of IDH1 ${ }^{\text {mut }}$ led to a marked reduction in JJ012 capacity for anchorage-independent growth in soft agar [22]. To verify whether HIF-1 $\alpha$ contributes to the observed promotion of colony formation by IDH mutation, we performed this assay under conditions of hypoxia ( $1 \%$ $\left.\mathrm{O}_{2}\right)$ and normoxia $\left(21 \% \mathrm{O}_{2}\right)$ using JJ012 parental and IDH1${ }^{\mathrm{m}} \mathrm{K}$ KO cells. We observed that hypoxia caused a dramatic increase in colony numbers in the IDH $1{ }^{\text {mut }} \mathrm{KO}$ groups (over $60 \%$; $p<0.05$ ), compared with those grown under normoxic conditions (Figure 4(a)). A similar pattern was also seen in the parental cells (Figure 4(a)). Notably, growth in hypoxia appeared to abolish the previously reported difference in colony formation between the parental and IDH1 ${ }^{\text {mut }} \mathrm{KO}$ JJ012 cells performed in a normoxic atmosphere (22). HIF- $1 \alpha$ levels under both conditions were analyzed by immunoblotting. As expected, incubation with $1 \% \mathrm{O}_{2}$ stimulated HIF- $1 \alpha$ expression to comparable levels in all three cell groups (Figure 4(b)). Together, these results suggest that IDH1 mutation contributes to JJ012 cells oncogenic functions, at least in part through HIF- $1 \alpha$ activation. 


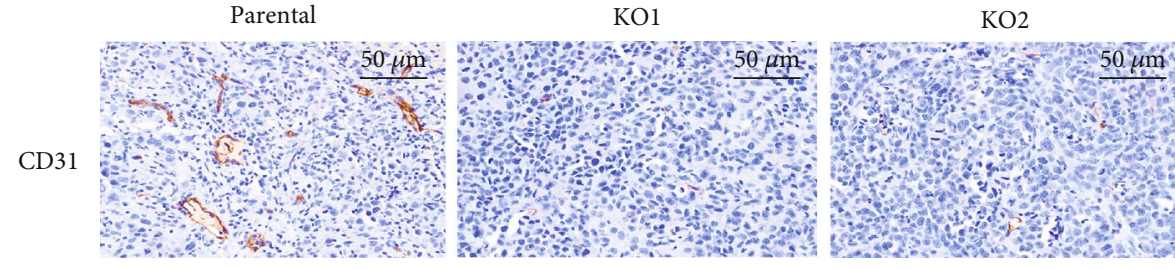

(a)
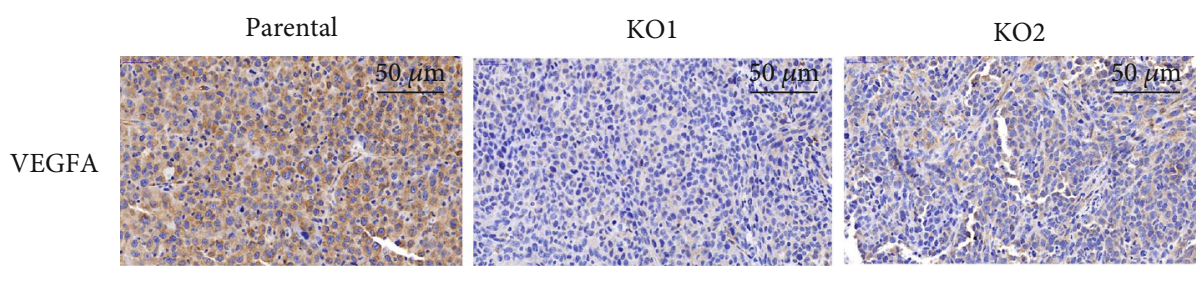

(b)
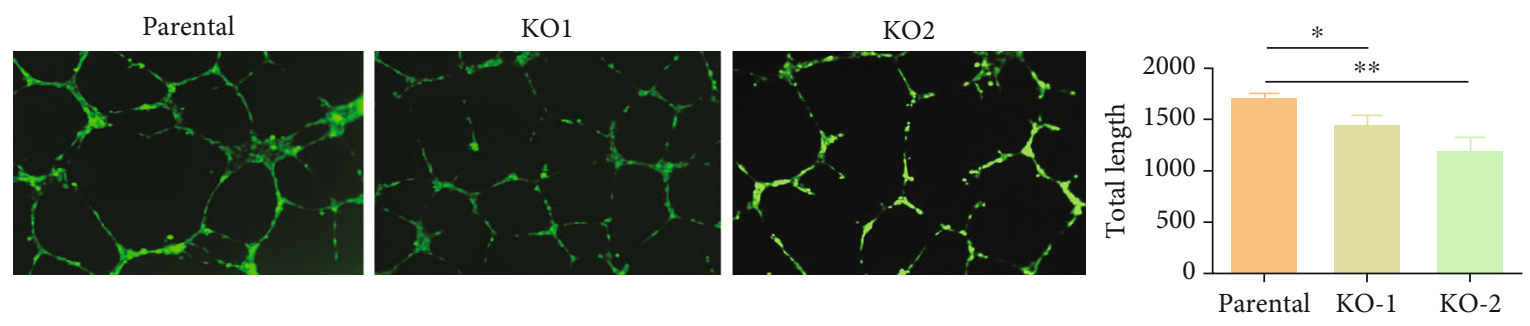

(c)
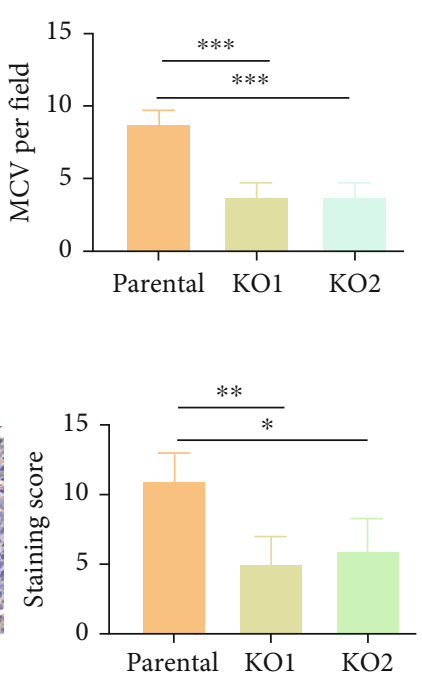

Parental KO1 KO2
) 

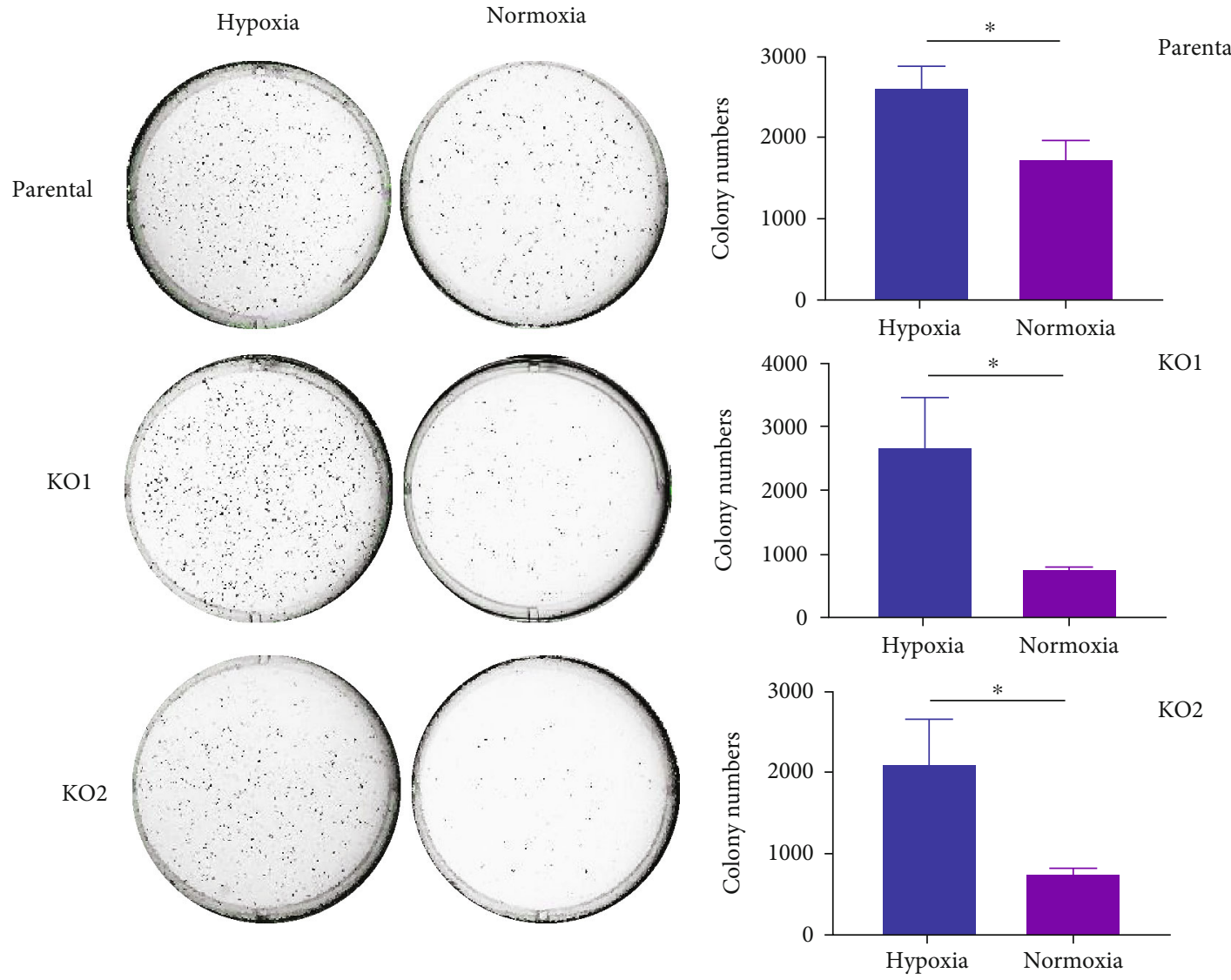

(a)

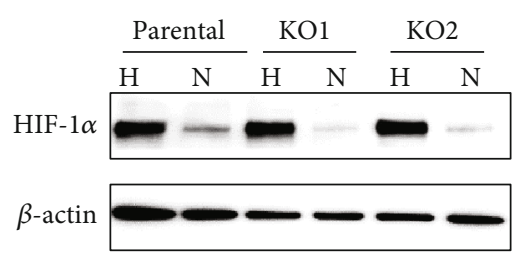

(b)

FIGURE 4: HIF- $1 \alpha$ contributes to the tumorigenic function of IDH1 mutation in JJ012 cells. (a) Colony formation assay with JJ012 parental and IDH1 ${ }^{\text {mut }} \mathrm{KO}$ cells under hypoxic $\left(1 \% \mathrm{O}_{2}\right)$ and normoxic $\left(21 \% \mathrm{O}_{2}\right)$ conditions. $5 \times 10^{3}$ cells per well were seeded in 6-well plates and incubated for 10-14 days. Graphs compare effects of hypoxia and normoxia on the number of colonies in the parental, KO1, and KO2 groups. Data indicate mean \pm SEM of triplicate cultures and are representative of 3 independent experiments. $* p<0.05$. (b) Immunoblot shows HIF- $1 \alpha$ levels in JJ012 parental and IDH1 ${ }^{\text {mut }} \mathrm{KO}$ cells under normoxia $(\mathrm{N})$ and hypoxia $(\mathrm{H})$ conditions.

that IDH1 ${ }^{\text {mut }}$ knockout cells formed less colonies than parental cells under normoxia condition, but exogenous induction of HIF- $1 \alpha$ significantly boosted the colonyforming capacity of these cells to a degree that is comparable with that of the parental cells. This suggests that activation of HIF- $1 \alpha$ signaling is involved in the tumorigenic activity of IDH $1^{\text {mut }}$ in vitro. Of note, our previous study has shown that loss of $\mathrm{IDH}^{\text {mut }}$ led to a marked attenuation of chondrosarcoma tumor formation and D$2 \mathrm{HG}$ production in a xenograft model [22]. Since anchorage-independent growth is tightly correlated with tumorigenic potential in vivo, it is conceivable that the attenuated HIF- $1 \alpha$ signaling caused by loss of IDH1 $1^{\text {mut }}$ might contribute to the observed inhibition of chondrosarcoma formation in the xenograft model.
Angiogenesis is a key contributor to tumor progression and metastasis. HIF- $1 \alpha$ and VEGF are known to play crucial roles in the tumor angiogenic process $[12,25,32]$. It is established that VEGF expression is mediated by HIF-1 $\alpha$ during hypoxia. The VEGF gene contains a number of HIF- $1 \alpha$ binding sites at its regulatory region, and HIF- $1 \alpha$ is able to activate the VEGF promoter [33, 34]. HIF- $1 \alpha$-induced VEGF expression is implicated in the angiogenic switch in chondrosarcoma [32]. Interestingly, studies have shown that IDH mutation is associated with elevated levels of HIF- $1 \alpha$ and VEGF levels in IDH-mutant gliomas [18, 35]. Consistently, we found the expression of angiogenic markers, VEGFA and CD31, was significantly reduced in the IDH1 $1^{\text {mut }}$ $\mathrm{KO}$ cell-derived tumors, suggesting that IDH1 mutation is associated with the angiogenic potential of chondrosarcoma 
cells. This association was solidified by in vitro vascular tube formation assay, which shows that the secretome of IDH $1^{\text {mut }}$ KO cells substantially reduced HUVECs' ability to from primitive vascular tubes in comparison with the secretome of parental cells. To be noted, our previous study also showed an association between IDH mutation and aberrant activation of integrin signaling in chondrosarcoma cells [22]. A few integrins have been implicated in blood vessel formation and regulation of cell growth, survival, and migration during tumor angiogenesis and metastasis $[36,37]$. Interestingly, although VEGF expression is induced by HIF- $1 \alpha$ during hypoxia [38], its expression can also be modulated by tumor integrins, resulting in efficient tumor angiogenesis under normoxic conditions [39]. Therefore, integrin and HIF- $1 \alpha$ signaling might intertwine with each other by shared mediators such as angiogenic factors in the tumor microenvironment, and thus both pathways contribute to the process of angiogenesis in IDH-mutant chondrosarcomas [12, 36]. In any case, the established association in this study renders antiangiogenic molecules appealing candidates for combinatorial regimens with $\mathrm{IDH} 1^{\text {mut }}$ inhibitors for advanced chondrosarcomas. The use of angiogenesis inhibitors has been used as an adjunct to other forms of therapy for preventing development of malignant neoplasms [40]. Preclinical studies have shown the benefits of targeting angiogenesis in chondrosarcomas [41]. Given the modest results with IDH $1^{\text {mut }}$ inhibitors in chondrosarcoma [42], future efforts to improve the efficacy of these compounds might benefit from emphasis on biology-driven therapeutic strategies to improve response rates in IDH-mutated chondrosarcomas.

The present study was limited by the use of only one conventional chondrosarcoma cell line. In fact, due to the rareness of this malignancy, very few chondrosarcoma cell lines are available worldwide. Thus, more chondrosarcoma cell lines, or patients' primary tumor cells, will be essential to further investigate and strengthen the concept explored in this study. Interestingly, we did not find a similar association between IDH mutation and HIF- $1 \alpha$ in an IDH1mutant fibrosarcoma cell line, HT1080, which was originally reported as a fibrosarcoma of bone, but is now considered to represent a dedifferentiated chondrosarcoma. We believe that the discrepancy is due to pathogenesis heterogeneity between the chondrosarcoma cell lines as conventional and dedifferentiated chondrosarcomas were shown to exhibit distinct biological behaviors and clinical characteristics. As described previously, differential biology was also observed within gliomas and leukemias with regards to the effects of IDH mutation on PHDs and HIF-1 $\alpha$ activity [18-21]. Thus, much work remains to better understand the biology to fully clarify these discrepancies and identify the appropriate patient populations for specific targeted therapies. Nonetheless, herein, we identified a strong correlation between HIF- $1 \alpha$ activation and IDH1 mutation status in chondrosarcoma cells. Furthermore, this study unravels one aspect of chondrosarcoma pathophysiology and provides insightful therapeutic possibilities such as combinatorial regimens of antiangiogenic agents with $\mathrm{IDH}_{1}{ }^{\mathrm{mut}}$ inhibitors for patients with advanced IDH mutated chondrosarcoma.

\section{Data Availability}

The experimental data used to support the findings of this study are included within the article.

\section{Conflicts of Interest}

The authors have declared that no conflict of interest exists.

\section{Authors' Contributions}

L.L., X.H., J.C.T., J.Y., and J.Y. conceived the experimental plan. X.H. and L.L. performed the experiments. All authors contributed to the data analysis. X.H., L.L., J.C.T., and J.E.E. wrote the manuscript. Xiaoyu $\mathrm{Hu}$ and Luyuan Li contributed equally to this work.

\section{Acknowledgments}

We thank Xiang-Xi Mike Xu' Laboratory, David Robbins' Laboratory, and Tan Ince's Laboratory for use of their equipment and technical support. We thank Fan Yang for the assistance in real-time PCR, Yuguang Ban for the assistance in RNA-Seq analysis, and Hongbiao Jing for the assistance with reviewing immunohistopathology of tumor samples. This work was supported by the Sylvester Comprehensive Cancer Center, University of Miami Miller School of Medicine. This work was supported by the following grants: NCI 1 P30 240139-01 CA grant, National Natural Science Foundation of China (Grant No. 81871895), Young Taishan Scholars and Academic Promotion Program of Shandong First Medical University (Grant No. 2019RC003).

\section{References}

[1] W. A. Chow, "Chondrosarcoma: biology, genetics, and epigenetics," F1000Research, vol. 7, p. 1826, 2018.

[2] J. C. Trent, A. E. Rosenberg, R. Pollock, and T. F. Delaney, Sarcomas: evidence-based diagnosis and management, Demos Medical Publishing, New York, NY, 2020.

[3] I. J. MacDonald, C. Y. Lin, S. J. Kuo, C. M. Su, and C. H. Tang, "An update on current and future treatment options for chondrosarcoma," Expert Review of Anticancer Therapy, vol. 19, no. 9, pp. 773-786, 2019.

[4] E. Nazeri, M. Gouran Savadkoohi, K. Majidzadeh-A, and R. Esmaeili, "Chondrosarcoma: An overview of clinical behavior, molecular mechanisms mediated drug resistance and potential therapeutic targets," Critical Reviews in Oncology/ Hematology, vol. 131, pp. 102-109, 2018.

[5] M. F. Amary, K. Bacsi, F. Maggiani et al., "IDH1andIDH2mutations are frequent events in central chondrosarcoma and central and periosteal chondromas but not in other mesenchymal tumours," The Journal of Pathology, vol. 224, no. 3, pp. 334-343, 2011.

[6] F. G. Schaap, P. J. French, and J. V. Bovee, "Mutations in the isocitrate dehydrogenase genes IDH1 and IDH2 in tumors," Advances in Anatomic Pathology, vol. 20, no. 1, pp. 32-38, 2013.

[7] L. Dang, S. Jin, and S. M. Su, "IDH mutations in glioma and acute myeloid leukemia," Trends in Molecular Medicine, vol. 16, no. 9, pp. 387-397, 2010. 
[8] L. Dang, D. W. White, S. Gross et al., "Cancer-associated IDH1 mutations produce 2-hydroxyglutarate," Nature, vol. 465, no. 7300, p. 966, 2010.

[9] P. S. Ward, J. Patel, D. R. Wise et al., "The Common Feature of Leukemia-Associated IDH1 and IDH2 Mutations Is a Neomorphic Enzyme Activity Converting $\alpha$-Ketoglutarate to 2Hydroxyglutarate," Cancer Cell, vol. 17, no. 3, pp. 225-234, 2010.

[10] L. Li, J. E. Eid, A. C. Paz, and J. C. Trent, "Metabolic enzymes in Sarcomagenesis: Progress toward biology and therapy," BioDrugs, vol. 31, no. 5, pp. 379-392, 2017.

[11] D. Ye, Y. Xiong, and K. L. Guan, "The mechanisms of IDH mutations in tumorigenesis," Cell Research, vol. 22, no. 7, pp. 1102-1104, 2012.

[12] J. W. Lee, S. H. Bae, J. W. Jeong, S. H. Kim, and K. W. Kim, "Hypoxia-inducible factor (HIF-1) $\alpha$ : its protein stability and biological functions," Experimental \& Molecular Medicine, vol. 36, no. 1, pp. 1-12, 2004.

[13] H. Semukunzi, D. Roy, H. Li et al., "IDH mutations associated impact on related cancer epidemiology and subsequent effect toward HIF-1 $\alpha$," Biomedicine \& Pharmacotherapy, vol. 89, pp. 805-811, 2017.

[14] P. Carmeliet, Y. Dor, J. M. Herbert et al., "Role of HIF- $1 \alpha$ in hypoxia-mediated apoptosis, cell proliferation and tumour angiogenesis," Nature, vol. 394, no. 6692, pp. 485-490, 1998.

[15] B. Keith, R. S. Johnson, and M. C. Simon, "HIFlalpha and HIF2alpha: sibling rivalry in hypoxic tumour growth and progression," Nature Reviews. Cancer, vol. 12, no. 1, pp. 9-22, 2011.

[16] J. H. Marxsen, P. Stengel, K. Doege et al., "Hypoxia-inducible factor-1 (HIF-1) promotes its degradation by induction of HIF- $\alpha$-prolyl-4-hydroxylases," Biochemical Journal, vol. 381, no. 3, pp. 761-767, 2004.

[17] S. Salceda and J. Caro, "Hypoxia-inducible factor 1 alpha (HIF-1 alpha) protein is rapidly degraded by the ubiquitinproteasome system under normoxic conditions - its stabilization by hypoxia depends on redox-induced changes," Journal of Biological Chemistry, vol. 272, no. 36, pp. 22642-22647, 1997.

[18] S. Zhao, Y. Lin, W. Xu et al., "Glioma-derived mutations inIDH1Dominantly inhibit IDH1 catalytic activity and induce HIF-1 $\alpha$," Science, vol. 324, no. 5924, pp. 261-265, 2009.

[19] W. Xu, H. Yang, Y. Liu et al., "Oncometabolite 2Hydroxyglutarate Is a Competitive Inhibitor of $\alpha$-Ketoglutarate-Dependent Dioxygenases," Cancer Cell, vol. 19, no. 1, pp. 17-30, 2011.

[20] P. Koivunen, S. Lee, C. G. Duncan et al., "Transformation by the (_R_)-enantiomer of 2-hydroxyglutarate linked to EGLN activation," Nature, vol. 483, no. 7390, pp. 484-488, 2012.

[21] J. A. Losman, R. E. Looper, P. Koivunen et al., "(R)-2-hydroxyglutarate is sufficient to promote leukemogenesis and its effects are reversible," Science, vol. 339, no. 6127, pp. 1621-1625, 2013.

[22] L. Y. Li, X. Y. Hu, J. E. Eid et al., "Mutant IDH1 depletion downregulates Integrins and Impairs Chondrosarcoma Growth," Cancers, vol. 12, no. 1, p. 141, 2020.

[23] T. D. Schmittgen and K. J. Livak, "Analyzing real-time PCR data by the comparative C(T) method," Nature Protocols, vol. 3, no. 6, pp. 1101-1108, 2008.

[24] L. Li, A. C. Paz, B. A. Wilky et al., "Treatment with a small molecule mutant IDH1 inhibitor suppresses tumorigenic activity and decreases production of the Oncometabolite 2Hydroxyglutarate in human chondrosarcoma cells," PLoS One, vol. 10, no. 9, article e0133813, 2015.

[25] Y. Z. Yang, M. J. Sun, L. H. Wang, and B. Jiao, "HIFs, angiogenesis, and cancer," Journal of Cellular Biochemistry, vol. 114, no. 5, pp. 967-974, 2013.

[26] S. M. Frisch and R. A. Screaton, "Anoikis mechanisms," Current Opinion in Cell Biology, vol. 13, no. 5, pp. 555-562, 2001.

[27] M. Sasaki, C. B. Knobbe, M. Itsumi et al., "D-2-hydroxyglutarate produced by mutant IDH1 perturbs collagen maturation and basement membrane function," Genes \& Development, vol. 26, no. 18, pp. 2038-2049, 2012.

[28] M. Nepal, H. Jung Choi, B. Y. Choi et al., "Anti-angiogenic and anti-tumor activity of Bavachinin by targeting hypoxia- inducible factor-1 $\alpha$," European Journal of Pharmacology, vol. 691, no. 1-3, pp. 28-37, 2012.

[29] H. Zhong, A. M. de Marzo, E. Laughner et al., "Overexpression of hypoxia-inducible factor 1alpha in common human cancers and their metastases," Cancer Research, vol. 59, no. 22, pp. 5830-5835, 1999.

[30] D. Luo, H. Ren, W. Zhang, H. Xian, K. Lian, and H. Liu, “Clinicopathological and prognostic value of hypoxia-inducible factor- $1 \alpha$ in patients with bone tumor: a systematic review and meta-analysis," Journal of Orthopaedic Surgery and Research, vol. 14 , no. 1, p. $56,2019$.

[31] T. Kubo, T. Sugita, S. Shimose, T. Matsuo, K. Arihiro, and M. Ochi, "Expression of hypoxia-inducible factor-1alpha and its relationship to tumour angiogenesis and cell proliferation in cartilage tumours," Journal of Bone and Joint Surgery. British Volume (London), vol. 90, no. 3, pp. 364-370, 2008.

[32] J. M. Fang, L. Yan, Y. Shing, and M. A. Moses, "HIF-1alphamediated up-regulation of vascular endothelial growth factor, independent of basic fibroblast growth factor, is important in the switch to the angiogenic phenotype during early tumorigenesis," Cancer Research, vol. 61, no. 15, pp. 5731-5735, 2001.

[33] D. T. Shima, M. Kuroki, U. Deutsch, Y. S. Ng, A. P. Adamis, and P. A. D'Amore, "The Mouse Gene for Vascular Endothelial Growth Factor:," The Journal of Biological Chemistry, vol. 271, no. 7, pp. 3877-3883, 1996.

[34] J. A. Forsythe, B. H. Jiang, N. V. Iyer et al., "Activation of vascular endothelial growth factor gene transcription by hypoxiainducible factor 1," Molecular and Cellular Biology, vol. 16, no. 9, pp. 4604-4613, 1996.

[35] C. Yalaza, H. Ak, M. S. Cagli, E. Ozgiray, S. Atay, and H. H. Aydin, "R132H mutation in IDH1 gene is associated with increased tumor HIF1-alpha and serum VEGF levels in primary glioblastoma Multiforme," Annals of Clinical and Laboratory Science, vol. 47, no. 3, pp. 362-364, 2017.

[36] C. J. Avraamides, B. Garmy-Susini, and J. A. Varner, "Integrins in angiogenesis and lymphangiogenesis," Nature Reviews. Cancer, vol. 8, no. 8, pp. 604-617, 2008.

[37] G. Serini, D. Valdembri, and F. Bussolino, "Integrins and angiogenesis: a sticky business," Experimental Cell Research, vol. 312, no. 5, pp. 651-658, 2006.

[38] M. Kunz and S. M. Ibrahim, "Molecular responses to hypoxia in tumor cells," Molecular Cancer, vol. 2, p. 23, 2003.

[39] M. Lorger, J. S. Krueger, M. O'Neal, K. Staflin, and B. FeldingHabermann, "Activation of tumor cell integrin v 3 controls angiogenesis and metastatic growth in the brain," Proceedings of the National Academy of Sciences of the United States of America, vol. 106, no. 26, pp. 10666-10671, 2009. 
[40] L. Yadav, N. Puri, V. Rastogi, P. Satpute, and V. Sharma, "Tumour angiogenesis and Angiogenic Inhibitors: A Review," Journal of clinical and diagnostic research: JCDR, vol. 9, no. 6, pp. XE01-XE05, 2015.

[41] S. Stacchiotti, S. Ferrari, A. Redondo et al., "Pazopanib for treatment of advanced extraskeletal myxoid chondrosarcoma: a multicentre, single-arm, phase 2 trial," The Lancet Oncology, vol. 20, no. 9, pp. 1252-1262, 2019.

[42] W. D. Tap, V. M. Villalobos, G. M. Cote et al., "Phase I study of the mutant IDH1 inhibitor Ivosidenib: safety and clinical activity in patients with advanced chondrosarcoma," Journal of Clinical Oncology, vol. 38, no. 15, pp. 1693-1701, 2020. 